\title{
Retrospective Comparison of Cardiac Testing and Results on Inpatients with Low Pretest Probability Compared with Moderate/High Pretest Probability for Coronary Artery Disease
}

Aaron Lear, MD, CAQ, Merritt Huber, MD, Amy Canada, MD, Jessica Robertson, BS, Evan Bosman, BA, and Stephen Zyzanski, PhD

Objective: To determine whether admission, and provocative stress testing of patients who have ruled out for acute coronary syndrome put patients with low-risk category for coronary artery disease (CAD) at risk for false-positive provocative stress testing and unnecessary coronary angiogram/imaging.

Methods: A retrospective chart review was performed on patients between 30 and 70 years old, with no pre-existing diagnosis of CAD, admitted to observation or inpatient status chest pain or related complaints. Included patients were categorized based on Duke Clinical Score for pretest probability for CAD into either low-risk group, or moderate/high-risk group. The inpatient course was compared including whether provocative stress testing was performed; results of stress testing; whether patients underwent further coronary imaging; and what the results of the further imaging showed.

Results: 543 patients were eligible: 305 low pretest probability, and 238 moderate/high pretest probability. No difference was found in rate of stress testing relative risk $(\mathrm{RR})=1.01(95 \% \mathrm{CI}, 0.852$ to $1.192 ; P=0$ ); rate of positive or equivocal stress tests between the 2 groups: $R R=0.653(95 \%$ CI, 0.415 to $1.028 ; P=.07$, ). Low-pretest-probability patients had a lower likelihood of positive coronary imaging after stress test, $R R=0.061(95 \% \mathrm{CI}, 0.004$ to $0.957 ; P=.001)$.

Conclusion: Follow-up provocative testing of all patients admitted/observed after emergency department presentation with chest pain is unlikely to find CAD in patients with low pretest probability. Testing all low-probability patients puts them at increased risk for unnecessary invasive confirmatory testing. Further prospective testing is needed to confirm these retrospective results. (J Am Board Fam Med 2018;31:219-225.)

Keywords: Coronary Artery Disease, Pretest Probability, Stress Test

Chest pain is the second leading cause of emergency department visits in the United States, resulting in over 7 million visits annually. ${ }^{1}$ After pre-

This article was externally peer reviewed.

Submitted 11 July 2017; revised 1 November 2017; accepted 3 November 2017.

From the Cleveland Clinic Akron General Family Medicine, Akron, OH (AL); Medical University of South Carolina, Charleston, SC (MH); CHI Franciscan Family Medicine, Tacoma, WA (AC); Northeast Ohio Medical University, Rootstown (JR); Cleveland Clinic Akron General, Akron (EB); Case Western Reserve Department of Family Medicine, Cleveland (SZ).

Funding: none.

Conflict of interest: none declared.

Corresponding author: Aaron Lear, MD, CAQ, Cleveland Clinic Akron General Family Medicine, 1 Akron General Avenue, Akron, OH 44307 (E-mail: leara@ccf.org). sentation to the emergency department, the decision making process for evaluation of patients at low risk of acute coronary syndrome (ACS) remains controversial, and much attention has been paid in recent years to the identification and further evaluation of these patients. ${ }^{2-5}$ This decision point revolves around the need for further evaluation of their chest pain including provocative stress testing, whether it is necessary at all, and whether it needs to happen while admitted to hospital or can take place at a later date on an outpatient basis. ${ }^{2-5}$

The American Heart Association (AHA) 2010 guideline recommends provocative stress testing to induce ischemia on patients with low-risk chest pain presenting to the emergency department after negative serial cardiac enzymes rule out the diag- 
nosis of $\mathrm{ACS}^{1}$. These recommendations address the use of clinical risk predictors such as tobacco use, hypertension, or dyslipidemia in conjunction with clinical judgment to guide the evaluation and management of the patient. This is recommended in a nonsystematized way, and there is little discussion of the role of pretest probability, or the potential risks of undergoing provocative testing despite often being very low risk for obstructive coronary artery disease (CAD). This is in contrast with the recommendations from the United Kingdom ${ }^{6}$ and the European Society of Cardiologists ${ }^{7}$, both of which comment on, and directly recommend evaluation of pretest probability before ordering provocative testing in those in low-risk categories for CAD. There are multiple validated pretest probability formulas available to assist with the determination of pretest probability, including the Duke score $^{8}$, the Diamond and Forrester score ${ }^{9}$, and a newer score developed in Europe ${ }^{10}$. All these tools may be used to assist in the pretest determination of probability of having symptomatic CAD.

In the United States, once patients at low risk for ACS are admitted for observation or to inpatient units, and determined not to have ACS, there is wide variability in the decisions made regarding provocative stress testing and further confirmatory imaging of coronary arteries. ${ }^{11-15}$ It is unclear what leads to this variability in decision making, and what affect this variability has on patient outcomes. Several recent studies suggest that it may be safe and appropriate to release patients considered to have low pretest probability for CAD from the hospital after it has been determined they are not having ACS without progressing to provocative stress testing. ${ }^{5,11-13}$

We hypothesized that admitting/observing patients and performing provocative testing on patients at low risk for ACS, who were also low pretest probability for obstructive CAD put them at risk for false-positive stress tests and unnecessary coronary angiography compared with those with a moderate or high pretest probability for CAD. We undertook a retrospective chart review of patients without known CAD admitted/observed for further evaluation and compared the hospital course of those in a low-risk group for CAD to those in a moderate/high-risk group. It is our goal to evaluate physician decision making about which patients receives provocative stress tests once admitted, and how this decision leads to further testing such as coronary angiogram; and further, to evaluate whether or not this is related to the pretest probability for CAD of the patient using.

\section{Methods \\ Design}

A retrospective chart review over 6 months' time between 2014 to 2015 at a 532 bed academic community hospital with an emergency department, a level 1 trauma center, and certified chest pain center was performed. Before initiation of the project, approval by the Institutional Research Review Board was obtained. A comparison of hospital course and outcomes for patients admitted for chest pain evaluation considered low risk for ACS. These patients were grouped by pretest probability status for CAD based on Duke Clinical Score ${ }^{8}$ into 2 separate groups 1) low risk for $\mathrm{CAD}$, or 2) moderate/high risk for CAD.

\section{Subjects}

Subjects were identified by a query of emergency department disposition database. Eligible patients were identified by their International Classification of Disease, version 9 (ICD-9) diagnosis code on being admitted to the hospital for further cardiac workup either with either inpatient or observation status. ICD-9 diagnoses of chest pain, chest pressure, chest heaviness, and chest pain-cardiac were identified for a 6-month period in 2014 to 2015. All patients reviewed had been determined to be low risk for ACS after undergoing history and physical examination; either a normal electrocardiogram (ECG), or an ECG with no change from a previous study; and a negative initial set of cardiac enzymes including troponin I while in the emergency department. The patients were then transferred to the inpatient unit under admission or observation status. Eligible subjects were those within the validated age range of the Duke Clinical Score ages 30 and 70 years who did not have an existing diagnosis of coronary artery disease. Figure 1 illustrates the progression of testing in this cohort of patients.

Exclusion criteria included ages outside of the Duke score validated age range of 30 to 70 years, existing $\mathrm{CAD}$, and admission to either the coronary or medical intensive care units, or a final diagnosis of ACS while admitted or under observation. This decision to exclude those who became positive for diagnosis of ACS once admitted was made with an eye toward inclusion of only those patients with no 
Figure 1. Flow diagram of included patients/results.

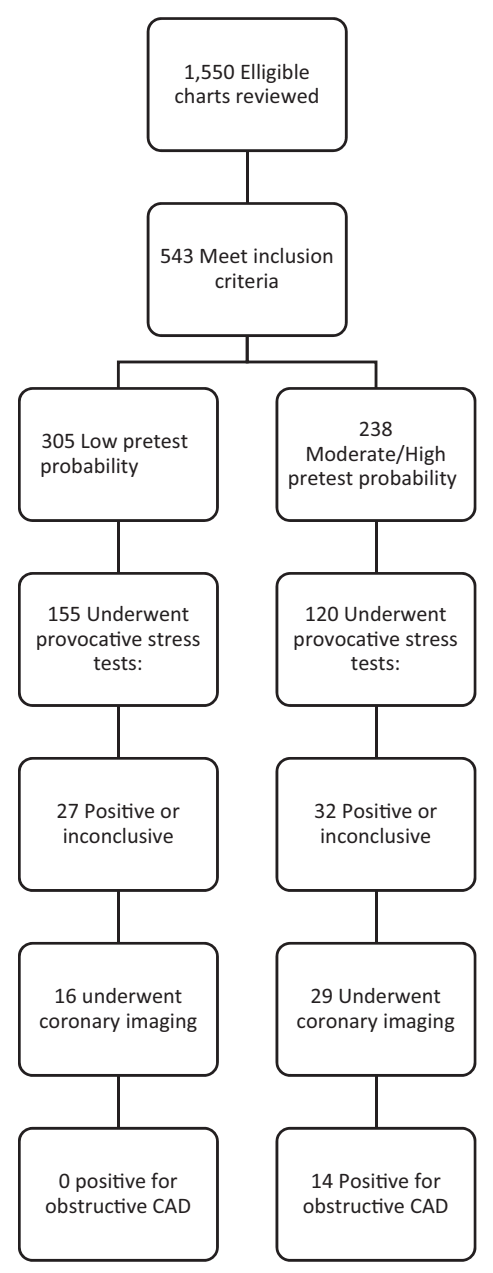

existing diagnosis of coronary artery disease (CAD).

\section{Data Collection and Analysis}

All original data were deidentified and recorded in a password protected Microsoft (Microsoft Corp, Redmond, WA) Excel 2013 spreadsheet. This database was later transformed into a coded file for evaluation with IBM SPSS statistics version 23 (IBM Inc, Armonk, NY).

The statistical analysis was done in 3 stages. Initially, descriptive statistics, including percentages and confidence intervals for demographic, CAD risk factors and outcome variables, were computed to provide a profile of the sample of patients studied. Descriptive statistics reported include the number of patients in low-risk and moderate/high-risk categories; the proportion of those in low-risk and moderate/high-risk category receiving provocative stress tests, the results of stress testing; and the results of coronary angiograms or computed tomography (CT) scans in those in the low and moderate/high-risk category. Next, inferential statistics specifically $\chi^{2}$ statistics, were used to analyze differences in patient demographics and CAD risk factors between the 2 Duke clinical score classifications. Lastly, $\chi^{2}$ statistics, exact statistics, and relative risk ratios were used in analyzing differences in patient outcomes between the 2 Duke clinical score classifications. Fisher's exact test was used to compare coronary angiography results in the low and moderate/high-risk groups due to the relatively small sample available for this comparison.

Primary outcomes were a comparison of the number of patients in low-risk versus moderate/high-risk category who received stress testing; a comparison of the results of stress testing and whether those in each group had a higher risk of positive/equivocal stress test; a comparison of the percentage of those in each group with obstructing CAD on angiography/CT at the level of $70 \%$ in at least a single artery, as defined by the Duke Clinical Score. After initial statistical evaluation, we performed post-hoc tests comparing the rates of those in each group with positive or equivocal stress tests who went on to further coronary imaging with CT scan or angiography.

\section{Results}

A total of 1550 patients were identified and had hospital charts reviewed; 544 of these met initial inclusion criteria. After retrospectively applying the Duke Clinical Score for pretest probability, 306 of 544 were low pretest probability for CAD. One of these patients was eventually excluded from the study when her serial troponin I elevated and she was diagnosed with ACS, leaving 305 patients in the low-risk category. The remaining 238 patients were in the moderate/high-risk group. Patients in the low-risk category were significantly more likely to be younger, female, and exhibit significantly lower rates of diabetes, hypertension, hypercholesterolemia, and smoking. Demographic and CAD risk-factor information is included in Table 1.

Both groups underwent provocative stress tests at a similar rate, and the relative risk of undergoing a stress test showed no statistical difference between groups 1.01 (95\% CI, 0.852 to $1.192 ; P=$ .93 by $\chi^{2}$ test). The proportion of positive or equivocal stress tests was lower in the low-risk group $(17 \%)$ compared with the moderate-to-high-risk 
Table 1. Demographics and Medical Conditions of Included Subjects

\begin{tabular}{|c|c|c|c|c|}
\hline Category & Low Risk n, (\% of Low Risk) & $\begin{array}{l}\text { Moderate/High Risk n, } \\
\text { (\% of Mod/High Risk) }\end{array}$ & $P$ Value* & $\begin{array}{l}\text { Total } \\
(\%)\end{array}$ \\
\hline 30 to 44 years & $98(32)$ & $24(10)$ & - & $122(22)$ \\
\hline 45 to 65 years & $190(62)$ & $190(80)$ & $.001^{\dagger}$ & $380(70)$ \\
\hline$>65$ years & $17(6)$ & $24(10)$ & - & $41(8)$ \\
\hline Male & $49(16)$ & $177(74)$ & .001 & $226(42)$ \\
\hline Diabetes & $37(12)$ & $94(40)$ & .001 & $131(24)$ \\
\hline $\mathrm{HTN}$ & $168(55)$ & $154(65)$ & .024 & $322(59)$ \\
\hline Hypercholesterolemia & $78(26)$ & $103(43)$ & .001 & $181(33)$ \\
\hline Obesity (BMI > 30) & $157(52)$ & $116(49)$ & .527 & $273(50)$ \\
\hline Smoker & $99(33)$ & $141(59)$ & .001 & $240(44)$ \\
\hline Total & 305 & 238 & & $543(100)$ \\
\hline
\end{tabular}

*All $P$ values are based on $\chi^{2}$ statistics.

${ }^{\dagger} P$ value for age applies to a difference between all age groups.

BMI, body mass index; HTN, hypertension.

group (27\%). Based on the observed relative risk of 0.65 , patients in the low-risk group had a $35 \%$ lower rate of a positive/equivocal stress test than patients in the moderate/high-risk group. There was, however, no statistical difference in the relative risk (0.653) of having a positive/equivocal stress test between the 2 groups (95\% CI, 0.415 to 1.028 ; $P=.07$, by $\chi^{2}$ ), details listed in Table 2 . A post-hoc power analysis suggests we were underpowered to detect a difference in this group, and that we would have required a sample size nearly 200 patients larger to detect a difference of $10 \%$ between groups.

A breakdown of the type of provocative stress tests performed for the 274 tested shows only 15 ECG maximal exercise tests (5\%) compared with 259 stress tests with imaging (95\%), details listed in Table 3.

A total of 45 imaging studies of the coronary arteries were performed on patients in both groups, only 3 were CT scans. Of the 27 patients in the low-risk group, 16 underwent further testing with coronary angiogram or CT (59\%). Of the $32 \mathrm{pa}-$ tients in the moderate/high-risk group who had positive/equivocal stress tests, 29 underwent further testing with coronary angiogram or CT (91\%). A statistically significant difference between the likelihood of moving on to coronary imaging after a positive stress test for the 2 groups was found in post-hoc testing by $\chi^{2}(P=.005)$.

Of the 16 patients in the low-risk group who progressed to coronary angiogram or CT scan, none had obstructive CAD. Of the 29 patients in the moderate/high-risk group who progressed to coronary imaging, 14 (48\%) had a study positive for obstructive CAD. The relative risk of an angiogram positive for obstructive CAD in the low-risk group compared with the moderate/high-risk group was 0.061 (95\% CI, 0.004 to 0.957 ), and this difference was significant by Fisher's exact test $(P=$ .001). Thus, patients in the low-risk group had a $94 \%$ lower rate of a positive angiogram than patients in the moderate/high-risk group.

\section{Discussion}

Our findings confirm our hypothesis that patients with no previous diagnosis of $\mathrm{CAD}$, admitted to observation or inpatient status for serial cardiac enzymes and further evaluation of their chest pain may be at increased risk for unnecessary coronary angiogram. In our retrospective analysis, we found this to be true with patients determined to have a low pretest probability for CAD by the Duke score ${ }^{8}$ when comparing them with patients in the moderate/high-risk category. Our data suggest that those patients in the low-probability category with positive/equivocal stress test results are likely to be false-positive results, and in fact in our data set, every patient that underwent coronary imaging with angiogram or CT showed no obstructive CAD.

These data add to previous research published by Penumetsa et $\mathrm{al}^{11}$, which demonstrated the low yield of provocative testing of low-pretest-probability patients when using the Diamond and Forrester ${ }^{9}$ prediction model, an earlier iteration of the Duke score ${ }^{8}$ used in this project. There are significant differences in the 2 data sets, with $70 \%$ of all patients reviewed by 
Table 2. Low Pretest probability vs. Moderate/High Pretest Probability Subjects

\begin{tabular}{|c|c|c|c|c|c|}
\hline & $\begin{array}{l}\text { Low Pretest } \\
\text { Probability }\end{array}$ & $\begin{array}{l}\text { Moderate/ } \\
\text { High Pretest } \\
\text { Probability }\end{array}$ & Total & $\begin{array}{l}\mathrm{RR}^{*} \text { Low } / \mathrm{MH} \\
\quad(95 \% \mathrm{CI})\end{array}$ & $P$ Value \\
\hline Stress tested (\%) & $155 / 305(51 \%)$ & $120 / 238(50 \%)$ & $275 / 543(51 \%)$ & $1.01(0.852,1.192)$ & $.93^{\dagger}$ \\
\hline $\begin{array}{l}\text { Positive or equivocal stress } \\
\text { tests/stress tested }(\%)\end{array}$ & $27 / 155(17 \%)$ & $32 / 120(27 \%)$ & $59 / 275(21 \%)$ & $0.653(0.415,1.028)$ & $.07^{\dagger}$ \\
\hline $\begin{array}{l}\text { Positive coronary } \\
\text { angiogram or CT/ } \\
\text { positive or inconclusive } \\
\text { stress test }(\%)\end{array}$ & $0 / 16(0 \%)$ & $14 / 29(48 \%)$ & $14 / 45(31 \%)$ & $0.061(0.004,0.957)$ & $.001+$ \\
\hline
\end{tabular}

Testing results by Duke clinical score pretest probability category.

*Relative risk of low pretest probability category compared to moderate/high pretest probability group.

${ }^{+} P$-value based on $\chi^{2}$.

$+P$-value based on Fisher's exact test.

CI, confidence interval; CT, computed tomography.

Penumetsa et $\mathrm{al}^{11}$ undergoing stress testing, while approximately $50 \%$ overall of our patients undergoing testing. In neither data set was there an association with pretest probability and whether a stress test was ordered. It is also notable that our data set showed a very small amount of exercise stress tests being ordered overall, which may be a way to decrease falsepositive rates, especially in the low-pretest probability category. ${ }^{1}$ In a population where a large proportion of patients are tested, such as in the Penumetsa data, it would suggest a propensity to test most or all patients after the decision from the emergency department to observe for ACS. However, in our data set, the testing of only $50 \%$ of patients across all pretest probabilities suggests a haphazard approach to testing as one would expect increasing rates of testing in patients with higher risk, hopefully just based on physician discretion if not a validated prediction score. In a study with a similar methodology, Napoli ${ }^{13}$ reported a stress test rate of $49 \%$, similar to our data, but did show an association between stress rate and pretest probability category.

There is further discrepancy in published data on decision making when moving from positive/ equivocal stress results to confirmatory imaging.
Our data showed an overall conversion from positive or equivocal stress result to further imaging at an overall rate of $76 \%$. The probability of a true positive stress test seems to have played a role in decision making to move on to further testing in our cohort, with $59 \%$ of the low-risk group moving on to coronary imaging, compared with $91 \%$ of the moderate or high-risk group, which was a statistically significant difference $(P=.005)$. Hermann et $\mathrm{al}^{12}$ showed an overall rate of $26 \%$ of positive stress tests moving on to angiogram. In the very-low- and low-pretest probability categories, only 7 of 32 (22\%) went to angiography, with 3 being positive. The data of Napoli et $\mathrm{al}^{13}$ showed that despite 29 positive stress tests out of 1758 patients, 48 of the 1758 patients actually went on to angiography, suggesting the possibility that 19 patients went straight to coronary imaging, or went after a negative stress test. Napoli ${ }^{13}$ also reported, similar to our study, that all patients undergoing angiography in the low-pretest-probability category had negative findings. Penumetsa et $\mathrm{al}^{11}$ reported that of the 89 positive stress tests, $23(13 \%)$ went on to further coronary imaging. Khare et $\mathrm{al}^{4}$ showed a rate of conversion to coronary

Table 3. Stress Test Type

\begin{tabular}{lccrr}
\hline Probability Category & No Stress Test (\%) & ECG Maximal (\%) & Stress with Imaging $(\%)^{\dagger}$ & Total (\%) \\
\hline Low pretest probability & $151(50)$ & $10(3)$ & $144(47)$ & $305(100)$ \\
Mod/high pretest probability & $118(50)$ & $5(2)$ & $115(48)$ & $238(100)$ \\
Total & $269(50)$ & $15(3)$ & $259(47)$ & $543(100)$ \\
\hline
\end{tabular}

Provocative stress tests type by pretest probability.

*ECG maximal: Treadmill stress test with ECG monitor-no imaging associated.

${ }^{+}$Stress with imaging: Includes both exercise and chemical stress test with echocardiography or nuclear imaging.

ECG, electrocardiogram. 
angiogram of $54 \%(59 / 110)$, and of the 59 angiograms performed, 41 were negative.

In recent years, multiple authors have published articles with similar topics to ours, but focusing on slightly different aspects of the emergency department and hospital evaluation of low-risk chest-pain patients including the utility of admitting and testing these patients ${ }^{12,15}$; the safety of rapid discharge after initial ECG and cardiac enzyme testing ${ }^{16}$; the identification of low-risk patients and potential early discharge of these patients ${ }^{17}$; the effect on length of stay and cost of chest pain units, and rapid discharge. ${ }^{4,3}$ Our project was initiated due to the risk we believe patients undergo when being stress tested indiscriminately. We believe that when patients at low risk for $\mathrm{CAD}$ based on a validated model, the Duke score in this case, the likelihood is that positive results are falsely positive. This sentiment has been demonstrated by Ladapo et $\mathrm{al}^{18}$ who reported on the rise in outpatient stress testing ordered, and particularly stress testing with imaging suggesting that up to $30 \%$ of stress tests with imaging, and 14\% of exercise stress tests were ordered inappropriately in the years 2005 to 2010. As is true in other referenced articles, the authors comment on the unnecessary utilization of medical resources and dollars, but not the risks it poses to patients of the consequences of falsely positive results.

Once a positive test is found, a decision must be made on how to interpret it, and what further steps are appropriate. In the case of provocative stress testing, this often leads to coronary angiogram. This procedure is generally considered safe and seems to be becoming more so. The historic major complication rate of approximately $1 \%$ to $2 \%{ }^{19}$ but in recent years seems to have declined below $1 \%{ }^{20}$ It does however carry risk for several major adverse outcomes including death, myocardial infarction, stroke, dye-induced nephropathy ${ }^{19,20}$, among others, and if it can be avoided by using pretest probability to influence who is tested, we believe this would be in the best interest of patients.

As in other similar articles, we believe that our data show that there is likely a large proportion of patients who may safely be discharged from the emergency department, observation, or inpatient unit without provocative testing. The preponderance of this data suggests the safety of a conservative protocol that delays or defers provocative testing on patients who fit a profile of low-pretest probability, or perhaps referring patients back to primary care practitioners to make decisions based on risks and benefits of further testing with patients with whom they are familiar. When taken in the context of recent evidence regarding evaluating low-risk chest pain patients, it is appropriate to not only question whether the current guidelines are appropriate, but whether they are safe. There is inherent risk for false-positive results when testing populations with low disease prevalence. ${ }^{21}$ Those patients falling into the low-risk category of various $\mathrm{CAD}$ pretest probabilities make up a population with low prevalence of disease. Continued blanket recommendations for provocative stress testing of all low-risk patients admitted for observation or inpatient evaluation for ACS has the risk of subjecting millions of patients annually to a confirmatory procedure with significant risk, especially if the screening test leading the angiogram is likely to be a false positive at its outset.

We believe these findings speak to the implications of national guidelines, which pay attention to the benefit of testing, and diagnosis, and pay little heed to risks inherent in testing those that have little risk for disease.

\section{Limitations}

Limitations to our study include the retrospective nature of the study. We also did not review medical records before or after our 6-month period, which may give more information as to why the rate of stress testing was surprisingly low. It is also a study taking place in a single center, and may speak more to the practice style and culture within this center, compared with other regional or national hospital systems. We also did not evaluate the decision making process for moving on to coronary angiogram after positive or indeterminate stress testing, which may have helped explain the different rates of performing coronary angiograms. Finally, when comparing the rates of those undergoing coronary imaging, the sample sizes were relatively small and our outcome rates would be expected to vary in other studies with larger sample sizes.

\section{Conclusion}

Consistent with other recent data ${ }^{1-13}$, our results suggest that provocative testing of low-risk chest pain patients considered low risk for $\mathrm{CAD}$ is unlikely to unearth obstructive CAD, but can subject these patients to unnecessary invasive testing with the possibility of serious complications. Further, the rate at which these low-risk patients with positive or equiv- 
ocal stress testing went on to confirmatory coronary angiogram (59\%) suggests that the physicians taking care of the patients recognize that stress test abnormalities are often not indicative of true CAD. Even with this decreased rate of confirmatory testing, compared with the intermediate and high-risk group, all those low-risk patients receiving coronary angiograms were negative for obstructive CAD.

We believe these findings speak to the implications of national guidelines, which pay attention to the benefit of testing and diagnosis, and pay little heed to risks inherent in testing those that have little risk for disease. We believe the mounting evidence calling into question the current blanket recommendations for provocative stress testing by the AHA should be revisited.

The authors thank the Cleveland Clinic Akron General Medical Center research department for technical and personnel support.

To see this article online, please go to: http://jabfm.org/content/ 31/2/219.full.

\section{References}

1. Amsterdam EA, Kirk JD, Bluemke DA, et al. Testing of low-risk patients presenting to the emergency department with chest pain: A scientific statement from the American Heart Association. Circulation 2010;122:1756-76.

2. Scott AC, O'Dwyer KM, Cullen L, Brown A, Denaro C, Parsonage W. Implementation of a chest pain management service improves patient care and reduces length of stay. Crit Pathw Cardiol 2014;13:9-13.

3. Kline JA, Jones AE, Shapiro NI, et al. Multicenter, randomized trial of quantitative pretest probability to reduce unnecessary medical radiation exposure in emergency department patients with chest pain and dyspnea. Circ Cardiovasc Imaging 2014;7:66-73.

4. Khare RK, Powell ES, Venkatesh AK, Courtney DM. Diagnostic uncertainty and costs associated with current emergency department evaluation of low risk chest pain. Crit Pathw Cardiol 2008;7:191-6.

5. Mahler SA, Burke GL, Goff DC, et al. Avoidable utilization of the chest pain observation unit: Evaluation of very-low-risk patients. Crit Pathw Cardiol 2013;12:59-64.

6. Skinner JS, Smeeth L, Kendall JM, et al. NICE guidance. Chest pain of recent onset: assessment and diagnosis of recent onset chest pain or discomfort of suspected cardiac origin. Heart 2010;96:974-8.

7. Montalescot G, Sechtem U, Achenbach S, et al. 2013 ESC guidelines on the management of stable coronary artery disease: The Task Force on the management of stable coronary artery disease of the European Society of Cardiology. Eur Heart J 2013;34:2949-3003.
8. Pryor DB, Harrell FE Jr, Lee KL, Califf RM, Rosati RA. Estimating the likelihood of significant coronary artery disease. Am J Med 1983;75:771-80.

9. Diamond GA, Forrester JS. Analysis of probability as an aid in the clinical diagnosis of coronary-artery disease. N Engl J Med 1979;300:1350-8.

10. Genders TS, Steyerberg EW, Hunink MG, et al. Prediction model to estimate presence of coronary artery disease: retrospective pooled analysis of existing cohorts. BMJ 2012;344(June):e3485.

11. Penumetsa SC, Mallidi J, Friderici JL, Hiser W, Rothberg MB. Outcomes of patients admitted for observation of chest pain. Arch Intern Med 2012;172:873-7.

12. Hermann LK, Newman DH, Pleasant WA, et al. Yield of routine provocative cardiac testing among patients in an emergency department-based chest pain unit. JAMA Intern Med 2013;173:1128-33.

13. Napoli AM. The association between pretest probability of coronary artery disease and stress test utilization and outcomes in a chest pain observation unit. Acad Emerg Med 2014;21:401-7.

14. Demarco DC, Papachristidis A, Roper D, et al. Pretest probability risk scores and their use in contemporary management of patients with chest pain: One year stress echo cohort study. JRSM Open 2015; 6(11):2054270415611295.

15. Cotarlan V, Ho D, Pineda J, Qureshi A, Shirani J. Impact of clinical predictors and routine coronary artery disease testing on outcome of patients admitted to chest pain decision unit. Clin Cardiol 2014; 37:146-51.

16. Than M, Aldous S, Lord SJ, et al. A 2-hour diagnostic protocol for possible cardiac chest pain in the emergency department: A randomized clinical trial. JAMA Intern Med 2014;174:51-8.

17. Scott SR, Riedinger MS, Conner L, et al. Practice guidelines and reminders to reduce duration of hospital stay for patients with chest pain. An interventional trial. Ann Intern Med 1994;120:257-63.

18. Ladapo JA, Blecker S, Douglas PS. Physician decision making and trends in the use of cardiac stress testing in the United States: An analysis of repeated cross-sectional data. Ann Intern Med 2014;161:482-90.

19. Scanlon PJ, Faxon DP, Audet A, et al. ACC/AHA guidelines for coronary angiography. A report of the American College of Cardiology/American Heart Association Task Force on practice guidelines (Committee on Coronary Angiography). Developed in collaboration with the Society for Cardiac Angiography and Interventions. J Am Coll Cardiol 1999 May;33:1756824.

20. Tavakol M, Ashraf S, Brener SJ. Risks and complications of coronary angiography: A comprehensive review. Glob J Health Sci 2012;4:65-93.

21. Baldessarini RJ, Finklestein S, Arana GW. The predictive power of diagnostic tests and the effect of prevalence of illness. Arch Gen Psychiatry 1983; 40:569-73. 